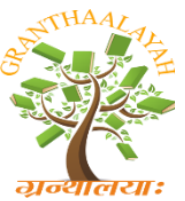

\author{
INTERNATIONAL JOURNAL OF RE
GRANTHAALAYAH \\ A knowledge Repository
}

Science

\title{
THE CHARACTERISTICS OF THE USE OF INSTAGRAM AMONG ADOLESCENT GIRLS
}

\author{
Anne Maryani ${ }^{* 1}$, O. Hasbiansyah ${ }^{2}$, Doddy Iskandar ${ }^{3}$, Maman Suherman ${ }^{4}$, Oji Kurniadi ${ }^{5}$ \\ ${ }^{1}$ Faculty of Communication, Universitas Islam Bandung, Indonesia
}

\begin{abstract}
Social media provides opportunities for users to express their thoughts and feelings through writing, pictures and videos. The rich features of this platform make Instagram the most preferred platform for adolescents, including teenagers in Indonesia. Various activities can be done through Instagram such as building friendships, seeking information and entertainment. The phenomenon of communicating online especially on Instagram can be observed through self-penetration theory that observes the closeness of relationships through communication intensity. Informants' preference for using Instagram gives them opportunities to build more intense relationships to meet their needs. The purpose of this study was to analyze the character of Instagram users on the activities of adolescent girls. This research was conducted using qualitative methods with a phenomenological approach. Data collection is done by in-depth interviews, observations and scientific references. The informants of this study were teenage girls in Bandung City and Regency who used Instagram for at least one year. The results showed that the activities of female teenage informants on Instagram were searching, stalking and posting. The meaning of Instagram for female teenage informants is a medium of self-expression that is displayed through text, images, videos, to socialize, to get entertainment from information provided by Instagram, is also a medium for expressing feelings. The awareness of young female informants in their activities on Instagram social media is already well related to the social implications resulting from the production and dissemination of messages on Instagram. So the informant planned in advance his message so as not to cause negative consequences that have legal consequences.
\end{abstract}

Keywords: Usage Characteristics; Adolescent Girls; Social Media; Instagram.

Cite This Article: Anne Maryani, O. Hasbiansyah, Doddy Iskandar, Maman Suherman, and Oji Kurniadi. (2019). "THE CHARACTERISTICS OF THE USE OF INSTAGRAM AMONG ADOLESCENT GIRLS." International Journal of Research - Granthaalayah, 7(10), 146-154. https://doi.org/10.29121/granthaalayah.v7.i10.2019.381.

\section{Introduction}

Social media provides opportunities for users to express their thoughts and feelings through writing, audio and visuals. Social media are the online communication Web sites and application channels dedicated to community-based input, interaction, content-sharing, and collaboration. 
Below are some broad categories for social media [1]. The rich features of this platform make Instagram the most preferred platform in Indonesia and its users are increasing at the world level of 1 billion as liputan6.com coverage. Instagram is the social network that has grown the most in recent years. In September 2017, it reached 800 million users according to the latest data according to the company itself. 59\% of users are between 18 and 29 years old, 33\% are between 30 and 49 . $60 \%$ have higher academic studies, and 63\%, almost one in three, earn more than $\$ 50,000$ per year, an important factor for any NGO [2] Instagram is widely used by young people because of its more dynamic features, and even though according to the survey users who dominate are male. The use of Instagram among women is interesting to explore. Social media re-contextualizes social constructions because people create new meaning out of the information they publish and receive in virtual social spaces [3].

Through social media, humans can express their desires or needs even though their use is inseparable from some positive and negative social implications. Facilities owned by social media platforms provide flexibility for users to create and show their identity. The large number of social media users in Indonesia has the potential to be exploited in various interests such as the economy, culture, politics and the images that appear on the Instagram platform page of attractive young women to be revealed to explore the representation of teenage girls through social media. Surveyed in Kuwait with women as samples are found in Middle Eastern countries, according to an empowerment map that describes where women have the most and least opportunities in the world. According to the global index of economic integration and empowerment, countries in the Middle East and Northern Africa region are ranked at the bottom on the economic potential of empowerment [4].

Through a phenomenological approach the writer reveals the awareness of adolescent girls when using social media Instagram. Several other studies focus on the identification of social roles and an investigation of their influence in online contexts [5]. Other research explores gendered surveillance on Instagram. The hashtag serves as an affordance across platforms, and this work expands on the literature of the rhetorical functions of hashtags [6]. Other research on Instagram examines the Results show that people who were not initially concerned about privacy are the most worried about the location-aware scenario; conversely, people who were initially connected are less worried about the location-aware scenario and find the scenario interesting. A deeper analysis of the results obtained allows us to draw guidelines that might be helpful to build an effective location-aware scenario [7]. Other studies To investigate the efficacy of using an "Instagram application" with a "home-exercise program" as a motivational stimulus in improving physical activity (PA) levels among female college students [8].

The absence of contextual clues related to internet users themselves clearly and reliably is the main difference that distinguishes communicating through online media with direct communication or face to face. As a result, online communication actors who do not use video call facilities cannot see the facial expressions, gestures, tone of voice, appearance, or physical of the person communicating so that it is more difficult to interpret their messages. The situation provides opportunities for internet users to present themselves differently from factual conditions or real situations outside the virtual world. Erving Goffman calls it Impression management, which sees interpersonal communication as a drama or play in which life is a communication situation built by the communication agent and the actor is the communicator who can display an impression that 
is in accordance with the wishes of the communication agent. As a participant in communication, the communicator is not only an actor but also a screenwriter who writes the manuscript "drama" of life. Goffman's self-presentation theory posits that an individual develops a sense of self by creating an impression to showcase to others in face-to-face environments. In such social interactions, individual controls which information to make available and how it should be made available, together form one's impression management strategy. Despite the offline origin of selfpresentation and related theories, researchers are expanding these purviews to online contexts. For instances, self-presentation is enhanced via computer-mediated-communication due to users exploiting technological aspects in certain online channels to manage their impressions [9].

The activity of planning the impression in the dramaturgy theory, can also be explained by the phenomenon explained in the Social Precense theory that the nature of interpersonal relationships is determined by the characteristics possessed by the participants, because communicating via the internet has a lack of non-verbal cues elements so the actors of communication on the internet are less get to know people with whom they communicate, so that it leads to less personal conversations [10]. Lack of adequate identity related to communication actors results in other communication actors paying less attention to the communication process because of their attention due to the absence of communication actors so that their attention can be diverted to other things that are more interesting. This condition results in a relationship that is less close because close relations are difficult to form and the discussion is more directed towards general matters.

The limitations revealed in dramaturgy theory and social presence can then be anticipated with a theory that assumes that caution in communication between internet users only occurs at the beginning of their communication. Furthermore, according to studies that have been developed, the time intensity factor plays an important role, namely by looking at the time intensity used in communicating online as assumed in social penetration theory. The intensity of adequate time used to communicate, turns out to be in line with the intensity of the time that is increasing and the impersonal aspect which is decreasing, so that the actors of communication exchange messages more. This assumption is as stated in Littlejohn's social penetration theory that we know others by "penetrating" the ball. The ball has breadth and depth. You can learn many different things about other people (breadth) and you can learn in depth information about one or two things (depth). As the relationship between two people develops, they share more aspects of themselves, adding depth and breadth to what they know [11]. Through these communication activities communication actors on the internet can develop and direct relationships between them in the desired direction as long as they have enough time to understand each other. This character enables communication actors in online media to get acquainted quickly because of the high intensity of communication between them.

The activity of planning the impression in the drama theory, can also be explained by the phenomenon explained in the Social Precense theory that the nature of interpersonal relationships is determined by the characteristics possessed by the participants, because communicating via the internet has a lack of non -verbal cues elements so the actors of communication on the internet are less get to know people with whom they communicate, so that it leads to less personal conversations [10]. Lack of adequate identity related to communication actors results in other communication actors paying less attention to the communication process because of their attention due to the absence of communication actors so that their attention can be diverted to other 
things that are more interesting. This condition results in a relationship that is less close because close relations are difficult to form and the discussion is more directed towards general matters.

The limitations revealed in dramaturgy theory and social presence can then be anticipated with a theory that assumes that caution in communication between internet users only occurs at the beginning of their communication. Furthermore, according to studies that have been developed, the time intensity factor plays an important role, namely by looking at the time intensity used in communicating online as assumed in social penetration theory. The intensity of adequate time is used to communicate, turns out to be in line with the intensity of the time that is increasing and the impersonal aspect which is decreasing, so that the actors of communication exchange messages more. This assumption is as stated in Littlejohn's social penetration theory that we know others by "penetrating" the ball. The ball has breadth and depth. You can learn many different things about other people (breadth) and you can learn in depth information about one or two things (depth). As the relationship between two people develops, they share more aspects of themselves, adding depth and breadth to what they know [11]. Through these communication activities communication actors on the internet can develop and direct relationships between them in the desired direction as long as they have enough time to understand each other. This character enables communication actors in online media to get acquainted quickly because of the high intensity of communication between them [12].

\section{Method}

The method used in this study is a qualitative research method with a phenomenological approach. Phenomenology was chosen as a method for this research study because phenomenology is about the study of essence through lived experience, and adolescent girls experiences are the focus of this research. The existential hermeneutic phenomenology of Merleau-Ponty was chosen because this method helps us come closest to understanding lived experience and essence [13]. Any behavior that appears on a new surface level can be understood or explained when it can uncover or reveal what is hidden in the world of consciousness or the world of knowledge of the human doer. Because, reality is actually subjective and meaningful. It depends on one's perception, understanding, and assumptions. The research subjects are female teenagers who use Instagram facilities and also other sources that can provide the widest possible information on matters relating to the research topic so that it can then be developed in generalizations. So that in the end the specificity that can be specified in the concoction of a unique context. Research subjects have the following criteria: 1. Teenage Girls who are active on Instagram, 2. At least 1 year active on Instagram. The object of this research is "Representation of Female Teenage Identity on Instagram". Instagram is a photo sharing application that allows users to take photos, apply digital filters, and share them to various social networking services, including Instagram owners. The main data collection techniques in phenomenology studies are in-depth interviews with research subjects, the completeness of the data can be deepened by using other techniques, such as participant observation, document search, and others. The data collected is primary data consisting of observations and interviews with key informants about communication activities on Instagram, and secondary data in the form of data obtained through the internet and references and documentation relating to communication activities of adolescent girls on Instagram. 


\section{Results and Discussions}

Instagram as one of the most popular social media teenagers because this platform has advantages compared to other social media. Through this platform informants can create digital photo albums through the activity of posting pictures, videos and textual content. Adolescent female informants often post pictures related to the events they experienced. When a sender chooses a medium for transmitting information to another person, he or she must consider a multitude of factors. These factors include the characteristics of the available media, environmental characteristics (such as the nature of the image and social factors), and the personal qualities of both the sender and the receiver [14].

The posted content is related to interesting personal experiences and is displayed through images such as self photos that aim to get a lot of comments and likes. Posting pictures and videos is preferable by female informants compared to text. Text content is rarely posted, only occasionally to comment on images posted by others. The activity of posting and sharing image, video, and text content is done for self-existence and to be part of the group of friends.

Image, video and text symbols for some female teenage informants do not have any special meaning, for other female teenage informants posted on instagram in the form of pictures, texts and videos only for the sake of hobbies, work through endorse products, and heartfelt. Message production on Instagram is made through message planning in advance for fear of offending others. Information becomes an important entity of social media because it creates a representation of its identity, produces content, and interacts [15]. The interesting thing from this study is that almost all female adolescent informants use Instagram as a medium for expressing their hearts through the display of images, videos and texts according to the situation felt and experienced. The asynchronous online communication system allows informants to plan in advance text messages, pictures and videos on Instagram.

All informants feel the need to plan the content that will be posted and disseminated on Instagram for fear that the message may lead to unwanted responses from other Instagram users. In pouring his thoughts and feelings the style of language used is honest as it is and reflects the character of its users who are still teenagers. The activity of posting and sharing messages on Instagram conducted by female teenage informants received mixed responses from other Instagram users, there were positive and negative responses. When obtaining a negative response the informant responds that it is not reactive for fear of being trapped in a prolonged conflict. Informants are aware of the legal consequences that arise due to conflicts that occur on social media and due to irresponsible posting. This cautious attitude shows the attitude of careful and planned informants in producing content.

Informants use Instagram for various needs, find various information for their needs which is also done through stalking, Instagram has a variety of functions as stated by Chase (2011), "Social media has a variety of functions that attract users, because it has several functions that can be utilized by users namely; conversation, building identity, grouping, and protecting reputation [16]. Sundar and Lamperos (2013) emphasized the function of internet technology media as Contemporary media technologies encompass the Internet, Websites, and an array of social media sites Facebook, Twitter, Tumblr, Instagram and Pinterest that allows for Inform the rapid creation 
and sharing of user-generated messages, as well as instantaneous communication with other uses on other platforms of hand-held devices [17]. Through Instagram, friend relationships can also be built so its existence is recognized and more maintained relations with other Instagram owner friends. If many of a person's friends are using a new means of communication, the person will be motivated to join in the communication and may learn how to use the medium through a combination of imitation and trial and error [14].

Another advantage of Instagram is that it can help informants to have a digital photo album that attracts informants in expressing and actualizing informants through the images, videos and writing available on Instagram. and in a sociocultural context it means that women are thought of and treated as sexual objects, which leads them to view and present themselves as such (StevensAubrey et al., 2009; Vandenbosch \& Eggermont, 2012). Hawkins, Richards, Granley, and Stein (2004), Hesse-Biber et al. (2006), Mills et al. (2002), and Owen and Spencer (2013) all confirmed the belief that women further present themselves the way the media represent the ideal body image. This action is somewhat pervasive, however, because people tend to seek affirmation by presenting themselves in a manner that is socially acceptable (Bessenoff, 2006; Fitzsimmons-Craft et al., 2012) [3].

The Instagram platform as a new communication technology product breeds new cultures and lifestyles for informants through informant activities in communicating, expressing through text, images and videos. The authors posit that ordinary conversation and extraordinary amplification methods share some consistencies with self-concept theory. "Self-concept" refers to the totality of an individual's thoughts and feelings having a reference to himself or himself as an object [9]. Instagram for informants is a necessity, so free time is used using Instagram for just stalking, chatting or searching. Generally, female teenage informants open Instagram every day, only each person has a different time intensity.

Digital multimedia technology is able to attract users to spend time to "sink" in the virtual world, social media for some speakers has become part of their lifestyle. The former approach views lifestyle as changing as an individual adapts to the surrounding environment while the later approach views life-style as an orientation which the individual wants to maintain by changing his or her actions. Changes in individual values, attitudes, and preferences may affect, in the long term, the individual's life-style as orientation [12].

Most of the time the informants focused on opening social media activities on Instagram, doing selfies, posting pictures and videos that they found interesting, peeking on other people's Instagram, shopping and finding information that suited their needs. The advantage of Instagram compared to other social media is the features that make it easy for users to develop and send image and video content. The main attraction of Instagram as social media is its function as a digital photo album about the lives of its users. Due to its rapid growth and exceptional reach, social net-working sites have become an integral part of many people's daily lives and a standard method of managing and constructing one's self-presentation. Many users choose to construct their online personas through photo selection on their profiles (Pempek et al. 2009; Salimkhan et al. 2010; Siibak 2009). These photos are not chosen at random; female users wish to present themselves as friendly and sociable and have been shown to regard presenting aesthetically pleasing photographs (eg, photos in which they themselves looked attractive, wore pleasing attire, 
posed in a beautiful setting) as more important than male users do (Manago et al. 2008; Siibak 2009). Female users additionally display more photos compared to male users (Lenhart and Madden 2007; Pempeket al. 2009) [18].

Selfie activities carried out by female teenage informants were then posted on Instagram, the posted selfies were the photos that they found most interesting with the aim of getting responses 'like' and comments. Selfie is a picture of yourself and spread through social media, there are several reasons for taking a selfie, namely: 1) the activity is a form of self-existence. Selfie is an attempt to self-representation on social media, an attempt to be considered to exist or exist in the network. Evans et al in call it a phenomenon of self-existence on social media. 2) Demonstrating open users, 3) to attract the impression of other users or users in the network of friends on social media [15].

Through selfie activities, female teenage informant's express emotions visually. Not only photos of yourself posted on Instagram, also photos of unique and interesting environments such as birthday moments, holiday moments, unique events. As defined by the Oxford English Dictionary, a selfie is a self-portrait photograph of one's own self (which may or may not be taken with others) captured using a camera or a camera phone. Although the selfie phenomenon was evident in an era where Polaroid cameras and Information Technology \& People [19]. Stalking activities or peeking other user profiles on Instagram is also carried out by female teenage informants. This activity is carried out based on the deep curiosity of the existence of other Instagram users. In addition, they also carry out activities to see the products they need such as clothing, etc. So to persuade users to follow social media, companies must provide relevant content such as information, entertainment, problem solving, customer service related to consumer interest in the content.

The meaning of Instagram for young female informants is not only for conversation but also business media so that many business people market their products on social media. The existence of social media for marketing activities is a tool that allows informants to provide value to the market continuously, even when you are not involved in a transaction. This value is delivered in the form of content. The present research begins to fill this voids by exploring the interplay of selfies, self-presentation, narcissism and self-concept on two popular visual content-sharing apps: Instagram and Snapchat. The authors examine how millennials use selfies to present their selfconcepts on visual content-sharing apps, in an effort to understand their attitudes and intentions to participate in selfie-marketing [9]. The role of informants in this environment is to provide prospects and customers with ongoing value by providing information (content) that solves their problems and meets brand needs [16].

Instagram for young women informants is also a medium of self-expression, which can facilitate them in expressing their thoughts and feelings through video, text and images. Unique, interesting events are produced and shared by informants through the activity of posting pictures, text, videos on Instagram so that for them Instagram is like a digital album of life that can be seen and responded to by other Instagram users at any time. The awareness to post secure content has grown so that a message is planned to be produced and posted. Because Instagram is also used as a communication medium to build friendships, build social relations and also for business purposes. 


\section{Conclusions}

Activities of female teenage informants in online media are searching, stalking and posting. Through the activity of reading and sending statuses, posting texts, pictures and videos, sending and commenting, liking, doing heartfelt. In addition, the informant also sought information about certain products. The meaning of Instagram for female teenage informants is a medium of selfexpression that is displayed through text, images, videos, to socialize, to get entertainment from information provided by Instagram, is also a medium for expressing feelings. The awareness of young female informants in their activities on Instagram social media is already well related to the social implications resulting from the production and dissemination of messages on Instagram. So the informant planned in advance his message so as not to cause negative consequences that have legal consequences.

\section{Acknowledgments}

This article was compiled from the research fundamentals of the Ministry of Research, Technology and Higher Education, we would like to thank reviewers who have corrected and completed this research, we also thank leaders who have facilitated, Rector of Universitas Islam Bandung, Chair of LPPM (Institute of Research and Community Service) Universitas Islam Bandung. The Dean of the Faculty of Communication Universitas Islam Bandung who has provided recommendations for this research.

\section{References}

[1] Media S. Connecting with Social Media: Communications \& Publicity. :57-60.

[2] Journal C. Artivism and NGO: Relationship between image and 'engagement' in Instagram. 2018;29-37.

[3] SELF-PRESENTATION AND SOCIAL MEDIA: A QUALITATIVE EXAMINATION OF THE USE OF INSTAGRAM BY AMATEUR NPC FEMALE FIGURE COMPETITORS A Thesis Presented to The Faculty of the Department of Kinesiology San José State University In Partial Fulfillment of the Requirements for the Degree Master of Arts by Victoria M. Lupinetti. 2015;(May).

[4] Riquelme HE. Instagram: its influence to psychologically empower women. 2018;31(6):1113-34.

[5] Balagué C, Ghassany M. The Identification and Influence of Social Roles. 2017;22(2007):337-62.

[6] Sebastian M. Article Instagram and Gendered Surveillance: Ways of Seeing the Hashtag. 2019; 17:40-5.

[7] Furini M, Tamanini V. Location privacy and public metadata in social media platforms: attitudes, behaviors and opinions. 2015;9795-825.

[8] Al-eisa E, Al-rushud A, Alghadir A, Anwer S, Al-harbi B, Al-sughaier N, et al. Effect of Motivation by (Instagram) on Adherence to Physical Activity among Female College Students. 2016;2016.

[9] Fox AK, Bacile TJ. Sel fi e-marketing: exploring narcissism and self-concept in visual usergenerated content on social media. 2018;1(July 2017):11-21.

[10] Baldwin, John R, Stephen D Perry MAM. Communication Theories. USA: Pearson Education Inc; 2004.

[11] Little John S dan KF. Teori Komunikasi. Jakarta: Salemba Komunika; 2009.

[12] Hasan S, Ukkusuri S V. Location Contexts of User Check-Ins to Model Urban Geo Life-Style Patterns. 2015;1-20. Available from: http://dx.doi.org/10.1371/journal.pone.0124819 
[13] Mottern R. Teacher-Student Relationships in Court-Mandated Adult Education: A Phenomenological Study. 2013;18:1-40.

[14] David R. Dunaetz, 1 Timothy C. Lisk 2 and Matthew Minsuk Shin3. Personality, Gender, and Age as Predictors of Media Richness Preference. Hindawi. 2015;

[15] Rulli Nasrullah. Media Sosial. Bandung: Simbiosa Rekatama Media; 2015. 132

[16] Chase L and KK. Social Media Sales Revolution. United States of Amerika: :Mc.Graw Hill; 2011.

[17] Perloff RM. Social Media Effects on Young Women's Body Image Concerns : Theoretical Perspectives and an Agenda for Research. 2014;363-77.

[18] Feltman CE, Szymanski DM. Instagram Use and Self-Objectification : The Roles of Internalization , Comparison , Appearance Commentary, and Feminism. Sex Roles [Internet]. 2018;311-24. Available from: http://dx.doi.org/10.1007/s11199-017-0796-1.

[19] Dey BL, Balmer JMT, Saren M. Selfie appropriation by young British South Asian adults cultural identity in social media. 2018;31(2):482-506.

\footnotetext{
*Corresponding author.

E-mail address: annemaryani0@gmail.com
} 